\title{
An analysis of the potential global impact of dosing regimen and rollout options for the ChAdOx1 $\mathrm{nCoV}$ - 19 vaccine
}

Ricardo Aguas

University of Oxford

Anouska Bharath

University of Oxford

Lisa White

University of Oxford https://orcid.org/0000-0002-6523-185X

\section{Bo Gao}

University of Oxford

Merryn Voysey

Oxford Vaccine Group, Department of Paediatrics, University of Oxford, United Kingdom

Andrew Pollard

University of Oxford https://orcid.org/0000-0001-7361-719X

Rima Shretta ( $\nabla$ rima.shretta@ndm.ox.ac.uk)

University of Oxford https://orcid.org/0000-0001-5011-5998

\section{Article}

Keywords: COVID-19, coronavirus, vaccine

Posted Date: March 11th, 2021

DOl: https://doi.org/10.21203/rs.3.rs-296726/v1

License: (c) (i) This work is licensed under a Creative Commons Attribution 4.0 International License.

Read Full License

Version of Record: A version of this preprint was published at Nature Communications on November 4th, 2021. See the published version at https://doi.org/10.1038/s41467-021-26449-8. 
An analysis of the potential global impact of dosing regimen and rollout options for the ChAdOx1 nCoV-19 vaccine

Ricardo Aguas ${ }^{1}$, Anouska Bharath ${ }^{1}$, Lisa J White ${ }^{1}$, Bo Gao ${ }^{1}$, Andrew J Pollard ${ }^{2}$, Merryn Voysey $^{2}$, Rima Shretta*1

${ }^{1}$ Nuffield Department of Medicine, University of Oxford: R. Aguas PhD, A. Barath PhD, L.J. White PhD, B. Gao PhD, R. Shretta PhD

${ }^{2}$ Oxford Vaccine Group, Department of Paediatrics, University of Oxford: A. J. Pollard FMedSci, M. Voysey DPhil.

*Corresponding author

Rima Shretta

Nuffield Department of Medicine

University of Oxford

Old Road Campus

Headington, Oxford OX3 7LF

United Kingdom

rima.shretta@ndm.ox.ac.uk 


\section{Summary}

\section{Background}

The ongoing COVID-19 pandemic has placed an unprecedented health and economic burden on countries at all levels of socioeconomic development, emphasizing the need to evaluate the most effective vaccination strategy in multiple, diverse environments. The high reported efficacy, low cost, and long shelf-life of the ChAdOx1 nCoV-19 vaccine positions it well for evaluation in different settings.

\section{Methods}

Using data from the ongoing ChAdOx1 nCoV-19 clinical trials, an individual-based model was constructed to predict the 6-month population-level impact of vaccine deployment. A detailed probabilistic sensitivity analysis (PSA) was developed to evaluate the importance of epidemiological, demographic, immunological, and logistical factors in determining vaccine effectiveness. Using representative countries, logistical plans for vaccination rollout at various levels of vaccine availability and delivery speed, conditional on vaccine efficacy profiles (efficacy of the booster dose, time interval between doses, and relative efficacy of the first dose) were explored.

\section{Findings and Interpretation}

Our results highlight how expedient vaccine delivery to high-risk groups is critical in mitigating COVID-19 disease and mortality. In scenarios where the number of vaccine doses available is insufficient for high-risk groups (those aged more than 65 years) to receive two vaccine doses, administration of a single dose of vaccine is optimal. This effect is consistent even when vaccine efficacy after one dose is just $75 \%$ of the levels achieved after two doses. These findings offer a nuanced perspective of the critical drivers of COVID-19 vaccination effectiveness and can inform optimal allocation strategies. These are relevant to highincome countries with a large high-risk group population as well as to low-income countries with younger populations, where the cost and logistical challenges of procuring and delivering two doses for each citizen represent a significant challenge. 


\section{Funding}

Bill and Melinda Gates Foundation (OPP1193472); Li Ka Shing Foundation; Oxford University COVID-19 Research Response Fund (ref: 0009280).

The funders played no role in the design or outcomes of this work.

\section{Contributors}

$R A, A B$, LW, and RS conceived the paper and contributed to the analysis. RA developed the model and wrote the code. BG ran the PSA and contributed to data processing. MV and AP provided the data, discussed the analysis, and commented on the draft. All authors have read, contributed to, and approved the final version of the manuscript.

\section{Declaration of interests}

The authors declare no conflict of interest.

\section{Data Sharing}

The data used to inform this analysis have been published and are available in the public domain. The code used is available at: https://github.com/ricardoaguas/como-ChAdOx1vaccine-.

\section{Acknowledgements}

LW is funded by the Li Ka Shing Foundation. RA is funded by the Bill and Melinda Gates Foundation (OPP1193472). The Covid-19 Modelling Consortium has support from the Oxford University COVID-19 Research Response Fund (ref: 0009280). The authors have not been paid to write this article by a pharmaceutical company or other agency. The authors are grateful to Adam Bodley for proofreading the final draft. 


\section{Introduction}

As of March $2^{\text {nd }} 2021$, almost 115 million people have been diagnosed with COVID-19 worldwide, and in excess of 2.5 million confirmed deaths have been reported ${ }^{2,3}$. Vaccination is a critical strategy to control the spread of SARS-CoV-2, the virus that causes COVID-19, and to reduce the severity of symptomatic disease. Three vaccines have already received emergency use authorization in the United Kingdom (UK). The developers of two of these vaccines have reported efficacies of 95\% for their vaccines in their respective Phase 3 trials (Pfizer/BioNTech and Moderna) ${ }^{4}$. The third vaccine, ChAdOx1 nCoV-19, jointly developed by Oxford University and AstraZeneca, demonstrated an acceptable safety profile and efficacy against symptomatic COVID-19, with no hospital admissions or severe cases of disease reported in the intervention arm during Phase 3 trials conducted in three countries. This vaccine can be stored and distributed at $2-8^{\circ} \mathrm{C}$ and will be made available at a lower cost than the other vaccines, making it suitable for global access, particularly in low- and middleincome countries (LMICs) $)^{5-7}$.

While clinical trials have validated the efficacy of the ChAdOx1 nCoV-19 vaccine in reducing symptomatic infection, appropriate national vaccination strategies across the world must consider heterogeneity among populations as well as the diverse demographic and socioeconomic environments of affected countries. In particular, the younger population typically present in LMICs justifies the need to assess the effects of associated behaviours and health profiles on vaccine effectiveness. These countries exhibit competing health, social, and economic challenges owing to inadequate healthcare infrastructure and a high prevalence of immunocompromising and infectious diseases. In these settings, individuals could also suffer complex vaccine responses when compared with responses in individuals in more developed economies ${ }^{8,9}$. At the same time, many LMICs have been unable to secure vaccine doses in advance from potential suppliers and thus are likely to have incomplete coverage of their populations, particularly in the short-term. The global COVID-19 vaccine alliance, COVAX, has pledged to procure and distribute vaccines equitably to LMICs; however, this will cover a maximum of $20 \%$ of the total population in each country ${ }^{10}$. Although the University of Oxford and AstraZeneca have made the largest supply commitment to LMICs at more affordable prices than other vaccine manufacturers, there is a need to evaluate the impact of a range of factors on vaccine effectiveness ${ }^{11-13}$. 
Due to shortages in supply, the UK government has instituted a policy of administering the booster dose of the vaccine at up to 12 weeks following the initial dose, prompting a debate among scientists, manufacturers, and governments on optimal dosing intervals for COVID19 vaccines ${ }^{14,15}$. The purpose of this analysis is therefore to evaluate the efficacy of the ChAdOx1 nCoV-19 vaccine in countries with different demographic profiles, as a function of vaccine efficacy, dosage regime (interval between initial and booster doses, or no booster at all), coverage, and immunity wane rate. Given the differences in healthcare infrastructure and vaccine access around the world, decision-makers should consider the effect of these factors on population-level impact to determine the most effective strategy for their context ${ }^{13}$.

Where vaccination programmes have begun, priority has so far been given to older age groups, individuals with co-morbidities, and frontline medical staff. The model developed therefore considers a simplified system where the vaccine is delivered to age groups in descending order while supplies are available. As there is limited evidence of indirect effects, that is, the potential for reductions in transmission, this vaccine effect was assumed to be negligible for the purposes of this analysis.

\section{Methods}

The methodology employed was very specifically tailored to the research question and its context. Vaccine production rates are always going to be insufficient to meet the demand generated by a global pandemic. In a context of limited vaccine dose availability, it is imperative to prioritize those individuals who would yield the greatest epidemiological benefit. Assuming the most pressing need is to reduce hospitalization rates and deaths, the initial targeting of those at higher risk for these outcomes seems logical, given that the alternative of immunizing sufficient people at lower risk for the indirect benefits to outweigh the direct benefits of a vaccine targeted at those at higher risk is not feasible with the number of vaccines available in the short-term. Even the UK, where mass production of the AztraZeneca vaccine has enabled $20 \%$ of the population to be vaccinated within 3 months, opted to prioritize the high-risk groups (those aged more than 65 years), partially because of the uncertainty around vaccine efficacy against infection. The ChAdOx1 nCoV-19 vaccine 
clinical trials were the only Phase 3 trials in which infection was evaluated as an outcome. No evidence was found for a transmission reduction effect (VE $=3.8 \%\left[-72.4\right.$ to 46.3]) ${ }^{7}$, but important questions were raised about how to allocate a limited number of doses to optimize the impact on symptomatic disease, given that a single-dose regimen could offer prolonged protection and thus a delay of the second dose could be warranted.

We began from the premise laid out above and implemented an individual-based, agedependent, static transmission model to predict the number of infections, clinical cases, and deaths expected to occur within 6 months of vaccination programme rollout. Individuals are simulated as autonomous systems, each with a set of attributes, informing their serostatus, vaccination uptake history (number of doses and dosing interval), and age. Box 1 details how the dynamics processes inherent to disease transmission and vaccination campaign logistics are considered in the model. 
Box 1. Consider a hypothetical scenario where the number of SARS-CoV-2 infections over 6 months in an unvaccinated population is 100,000 . Policymakers could opt for one of two alternative options with very different direct benefit outlooks when deciding on how to allocate the limited number of vaccine doses available to them.

Option 1: Vaccinate high contact groups (aged 25-40 years) (36\% of all infections, $10 \%$ of all hospitalizations, $1 \%$ of all deaths) ${ }^{1}$

Predicted infections:

$100,000 * 36 \% *$ [1-vaccine direct effect on infection ] $+100,000 * 64 \% *$ [1-vaccine indirect effect on infection]

Predicted hospitalizations:

Predicted infections*10\%*[1-vaccine direct effect on hospitalization] + Predicted infections * $90 \% *$ [ 1-vaccine Indirect effect on hospitalizations]

Predicted deaths:

Predicted infections*1\%*[1-vaccine direct effect on deaths] + Predicted infections*99\%*[ 1-vaccine indirect effect on deaths]

Option 2: Vaccinate high risk groups (aged $>65$ years) (10\% of all infections, $66 \%$ of all hospitalizations, $90 \%$ of all deaths) 1

Predicted infections:

$100,000 * 10 \% *[1$-vaccine direct effect on infection $]+100,000 * 90 \% *[1$-vaccine indirect effect on infection]

Predicted hospitalizations:

Predicted infections *66\%*[1-vaccine direct effect on hospitalization] + Predicted infections *34\%*[ 1-vaccine indirect effect on hospitalizations]

Predicted deaths:

Predicted infections*90\%*[1-vaccine direct effect on deaths] + Predicted infections*10\%*[ 1-vaccine indirect effect on deaths]

Thus, vaccines targeting high-contact groups would have to provide indirect effects in the order of $80 \%$ ( $80 \%$ reduction in risk in the untargeted population) to prevent an approximate number of deaths similar to that which would be provided by targeting the high-risk group with a direct vaccine effect against death of $85 \%$. 


\section{Transmission and clinical cascade}

The spread of COVID-19 is sensitive to the underlying network of contacts between infectious and susceptible individuals in their various societal spheres (home, work, public transport, etc). For a given population, we can summarize the number of contacts per day as an agedependent force of infection $\lambda(a)$, i.e. a daily risk of acquiring an infection given age $a$. The age-dependent risk of infection can then be defined as:

$$
\lambda(a)=k_{\lambda} \frac{\sum_{j=1}^{N} c_{i j} P_{j}}{\sum_{i=1}^{N}\left(\sum_{j=1}^{N} c_{i j} P_{j}\right)}
$$

Where $c_{i j}$ is the daily number of contacts between age groups $i$ and $j$ for a particular country, $P_{j}$ is the population age distribution, $N$ is the total number of age categories, and $k_{\lambda}$ is the overall daily risk of infection (which is informed by the number of infectious people in the population). Here, we decided to simplify the transmission process by making the daily risk of infection constant over time, thus having a static transmission model. When evaluating different epidemiological scenarios with different levels of population attack size over the 6month period explored here, we simply changed the daily risk of infection parameter until the correct attack rate was obtained. Without this constraint, computation of the very high number of simulations required to perform the sensitivity analyses presented here would be virtually impossible.

The risk of developing severe disease and possibly dying as a consequence of infection was informed by age-dependent infection hospitalization (IHR) and hospitalization fatality (HFR) ratios, published $\mathrm{in}^{16}$. Thus, the modelled daily risk that an individual will develop severe disease is given by $\lambda(a) \operatorname{IHR}(a)$, whereas the risk of dying is approximately $\lambda(a) \operatorname{IHR}(a) \operatorname{HFR}(a)$. The timing of these events and the lag between infection and clinical outcome are not relevant, as we are only making comparisons between synthetic populations, as detailed below.

\section{Vaccination delivery and vaccine efficacy}

Different vaccine dose allocation schemes were simulated, by limiting the number of doses distributed in 6 months, as well as allowing for different dosing intervals (delaying the second 
dose) and dosing splits (giving one dose vs two doses). The allocation of doses was always prioritized to the oldest age groups. Individuals were assigned vaccine doses in descending order of age until the maximum number of doses had been allocated.

Given a fixed number of available doses, one can calculate the target recipient population by looking at the dose-split proposal. If all doses are given as single doses in 6 months, then the target population for vaccination is equal to the number of available doses. At the other extreme, where all vaccinees receive two doses, the number of recipients would be half the number of available doses. Within the group that is meant to receive two doses of the vaccine, a $5 \%$ dropout rate (vaccine refusal) was imposed, and a range of booster dose intervals was explored.

We implemented three different logistical implementations of a vaccine campaign rollout: constant effort, frontloaded, and backloaded. The distinction was in the speed at which the target population received vaccine doses during the initial 2 months. As individuals were assigned a vaccine, the number of doses received would be determined by a draw from a uniform distribution according to the desired dose split. Individuals given two doses would be assigned a booster dose interval following a beta distribution with $\alpha=0.15$ and $\beta=0.95$.

Although vaccine efficacy was explored in the sensitivity analyses presented here, we centred the explored ranges around the point estimates presented $\mathrm{in}^{17}$. Vaccine efficacy, $V_{e}(t)$, was treated as a direct modulator of the risk of infection, clinical disease, and death; it was then defined for each individual, at each timestep of the simulation, as:

$$
V_{e}(t)=V_{i}^{j} e^{-\delta t}
$$

, where $V$ is the vaccine efficacy in an individual with baseline status $i$ that received dose number $j$ a $t$ number of days ago, while $\delta$ is the rate of loss of vaccine-induced immunity.

Throughout this paper, we present a sensitivity analysis of the post-dose two maximum efficacy, the relative efficacy of dose one vs dose two, and the booster dose interval. While doing so, we constrain vaccine efficacy against clinical disease to be the same as that against death, while vaccine efficacy against infection is fixed at $5 \%^{7}$. We also imposed a stepwise increase in post-dose two vaccine efficacy across an 8-week booster dose interval, as observed in the clinical trial ${ }^{17}$. This means that giving the second vaccine dose less than 8 
weeks after the first dose will result in a $25 \%$ lower post-dose two efficacy relative to the maximum assumed vaccine efficacy.

\section{Vaccine effectiveness}

The vaccination campaign population impact is referred to throughout as vaccine effectiveness and was defined as:

$$
V_{e f f}=100 \frac{A R_{v}-A R_{u}}{A R_{u}}
$$

, where $A R_{v}$ is the attack rate (over 6 months) in the vaccinated population, and $A R_{u}$ is the attack rate in a population that mirrors the vaccinated population in all aspects except vaccination. We thus have a pair of populations for each parameter set in our analyses and calculate the expected vaccine effectiveness for each parameter set as the relative difference in occurrence of each of the disease endpoints (infection, clinical symptoms, and death).

\section{Results}

We conducted an extensive initial sensitivity analysis to determine how the impact of rolling out a COVID-19 vaccination campaign in the UK depends on epidemiological, logistical, and immunological factors. The sensitivity of the modelled vaccine effectiveness to the variables explored is illustrated in Figure 1. It is clear that the prospects for vaccine impact are most sensitive to the number of vaccine doses available within 6 months, the speed of delivery within the same timeframe, and the vaccine efficacy (both the maximum efficacy post-dose two and the relative efficacy of dose one compared with dose two). Interestingly, for the same inputs, the median expected vaccine effectiveness is greater for deaths than it is for clinical cases.

From this first exploratory analysis one could immediately suggest that, for maximum effectiveness, a vaccine campaign should aim to vaccinate as many people as possible (thus governments/policymakers should procure the maximum number of doses possible), in the shortest time possible. These are by far the two variables the model outputs are most sensitive to, as can clearly be seen in Supplementary Figures 1 and 2 . These figures also reveal 
a very interesting interaction between the vaccine efficacy profile and an actionable decision of how the first available doses are to be distributed. In the frontloaded scenario, where the speed of vaccine delivery is maximal during the early stages of the 6-month vaccination campaign, a single-dose regimen is expected to perform significantly worse if vaccine efficacy post-dose one is $50 \%$ lower than vaccine efficacy post-dose two (top row). However, if the vaccine efficacy after both doses is the same (bottom row), a single-dose regimen can actually be preferable, especially if the number of vaccine doses available is small.

These initial results prompted further investigation of the possible interactions and trade-offs between the vaccine efficacy profiles and logistical implementation variables. In this detailed analysis, the population attack size was fixed at $12 \%$, delivery speed to frontloaded, and vaccine-induced protection to last 360 days. The results are summarized in Supplementary Figure 3. As determined by the initial sensitivity analysis, vaccine effectiveness is quite sensitive to the number of available doses, the maximum post-dose two efficacy, and the efficacy of the first dose relative to the second.

Two interesting results pertain to the sensitivity of the model to changes in the dose number split and the interval in days between doses for the two-dose regimen recipients. While giving everyone two doses, irrespective of all other variables, seems to be preferable to the singledose option, the length of the whiskers suggests there might be a parameter space for which the single-dose option is optimal, as seen in Supplementary Figures 1 and 2. Increasing the time interval between doses generally produces improved vaccine effectiveness, although a slight decrease in median effectiveness can be observed after an 8-week (56 day) interval. This is further investigated in Supplementary Figures 4 and 5, revealing an interesting tradeoff, with a large increase in predicted effectiveness after 7 to 8 weeks, followed by a small decrease as the booster dose interval expands, but only if the efficacy of the first dose is low. If the efficacy of the first dose is similar to the efficacy of the second, increasing the interval between doses up to 12 weeks does not decrease vaccine effectiveness.

Interestingly, we find a non-linear increase in effectiveness for large values of dose availability, which can be explained by the markedly non-linear risk of severe disease and death with age. As the number of available doses increases, a larger proportion of the population will receive a vaccine dose. However, since vaccines are allocated in descending order of age, as a larger proportion of the population is reached, more and more low-risk 
individuals are vaccinated, for whom the vaccine accrued benefits are smaller and smaller. It is then advisable to investigate these relationships for different settings.

We proceeded to investigate what factors could potentially influence the decision-making process regarding the distribution of doses during the first 6 months of vaccine programme rollout. We thus evaluated the relative predicted effectiveness of the single-dose versus the double-dose regimen, for different countries with potentially different dose availability, and assuming different vaccine efficacy profiles (Figure 2). For very high levels of dose allocation (high y-axis values), a two-dose regimen is clearly optimal. This starts to become less evident for scenarios where the protection conferred by the first dose gets closer to the post-dose two efficacy (moving right along the $x$-axis). Interestingly, when the number of available doses is small, the single-dose regimen will become more effective than the double-dose regimen, as the thick black line is crossed. The parameter combinations defining the line where there is a shift in strategy positioning are country dependent, with countries with older populations having a larger parameter space in which a single-dose option is preferable, as shown in Figure 3.

\section{Discussion}

The SARS-CoV-2/COVID-19 pandemic has created an unprecedented public health challenge, spurring a global race to develop and distribute viable vaccines. A vaccine that creates broad immunity against the SARS-CoV-2 virus could be the only effective means to control the pandemic and allow a return to "normalcy". To have a significant impact on the disease, a critical mass of the global population at risk will need to be vaccinated. However, many high-income countries have secured more than half of the available vaccine doses for themselves, leaving LMICs, which make up more than $85 \%$ of the global population, to find their own solutions ${ }^{18}$. To address the problem of equitable access, WHO, Gavi, and the Coalition for Epidemic Preparedness Innovations (CEPI) established COVAX, a global alliance that has pledged to pool investment and allocate and distribute COVID-19 vaccines equitably, particularly in LMICs ${ }^{10}$. However, COVAX is currently under-resourced and the doses secured are insufficient to achieve the coverage levels needed ${ }^{19}$. Supply constraints and new variants of SARS-CoV-2 are steering countries towards strategies that counter low 
access with dosing patterns or volumes to maximize the impact of the vaccines. Data from the ChAdOx1 nCoV-19 vaccine trials have allowed us to explore potential strategies to inform optimal allocation programmes, particularly in contexts where the cost and logistics of implementing multiple doses within a short timeframe may be challenging.

Our findings indicate that vaccine effectiveness is dependent upon (i) the country context, which includes the demographic profile, the attack rate of the virus, and the amount of vaccine that is available (which influences the proportion of the population that is vaccinated); ii) the characteristics of the vaccine, which include the efficacy of a single dose relative to a double dose and the waning of efficacy over time; and iii) the proportion of the population receiving the second dose, the time interval between doses, and the delivery speed.

Our analysis demonstrates that in scenarios where the number of vaccine doses available is insufficient for the highest risk groups (aged $>65$ years) to receive two doses, the allocation of a single vaccine dose is optimal. This effect is consistent even when the vaccine efficacy of a single dose is just $75 \%$ of the levels achieved after a double dose, until allocation drops to a population coverage of $10 \%$, after which vaccinating only the high-risk individuals, with two doses, is more effective. In scenarios where the number of doses available to the country is sufficiently high, or if the relative single-dose efficacy is low (50\% or less), providing a booster dose within 8 weeks would be preferable. Apart from these specific conditions, the results indicate that providing individuals with two doses of vaccine would have a similar effectiveness to the use of a single dose given to twice the number of individuals.

The speed at which the high-risk population is vaccinated greatly influences the expected vaccine effectiveness in preventing clinical cases and death. This is particularly true if the transmission rate is high, with faster vaccination reducing the number of infections in groups awaiting their first dose during the rollout. Distributing the vaccine very slowly provides an effectiveness of less than $10 \%$, regardless of the number of doses and allocations. The impact of allocation on outcomes is also greater when the population is vaccinated rapidly over a six-month period. In both of these scenarios, providing a single dose is preferable. 
An interesting trade-off was found between the booster dose interval and the relative vaccine efficacy of a single dose. For vaccines with large differences between first and second dose efficacy, delaying the booster dose interval past 8 weeks after the first dose was found to be detrimental. However, if a single dose provided at least $75 \%$ of the protection conferred by a double dose, delaying the booster dose interval to 12 weeks had a negligible impact on the number of cases and deaths. Given the similar reported efficacies of single and double doses of ChAdOx1 nCoV-19, a 12-week interval is the optimal scenario for this vaccine ${ }^{17}$. However, this finding may not be applicable to other COVID-19 vaccines.

These differences are more profound when considering the demographic characteristics of a population. In high-income countries, which have a larger older population (>65 years), a single-dose regimen will allow the vaccination of more individuals more quickly, with a correspondingly greater impact on cases and deaths. In the UK, the six-month allocation threshold above which a two-dose regimen would be preferred was found to be about $16.5 \%$.

The six-month allocation threshold above which a two-dose regimen would be preferred is much lower in LMICs, mainly due to mortality in the younger population. In these contexts, decision-makers will need to consider the affordability, availability, and logistical constraints and feasibility of implementing a single or a double dose, the dosage intervals, and delivery speed. Most LMICs lack the digital databases necessary to manage patient data, reliably track vaccine inventories, keep track of who has received which vaccine, and inform people where and when they are due for a booster. Governments would also need to ensure that they reserve sufficient stocks to allow the administration of booster doses. In these cases, a robust cost-benefit analysis of each option will need to be considered.

The dosing interval for COVID-19 vaccines has been a subject of debate among scientists, regulators, and governments around the world following the UK government's decision to prioritize administering the first dose of vaccine to as many at-risk people as possible and increasing the interval between the two doses to up to 12 weeks ${ }^{14,15,20}$. A one-dose vaccine regimen or a two-dose regimen with longer time intervals may be sufficient to reduce symptoms of COVID-19 in the most vulnerable individuals and ultimately slow the pandemic, given that the time difference between first and second doses was shown to have a negligible effect on overall vaccine effectiveness (clinical cases, infections, and 
deaths). Indeed, a recent WHO notification stated that some countries are facing "exceptional circumstances" and may want to delay second doses to "allow for a higher initial coverage". Other exceptional circumstances may involve trade-offs around the relative size of the highest risk population in a country and the currently unknown potential for a vaccine to reduce transmission, which may lead to some countries targeting highcontact groups to benefit from any potential indirect effects.

Nevertheless, these thresholds are likely to differ depending on the country context. For example, smaller countries may be able to rapidly rollout the vaccine to a higher percentage of their population compared with the speed at which larger countries can do this. It should be noted that an implementation strategy is determined at a country level. The assumptions made in this work are based on the association of certain parameters with the health infrastructure and existing population of certain country groups defined by income level.

Published clinical data were used to inform the parameters used in the model described in this paper. These data provide an aggregate efficacy of the ChAdOx1 nCoV-19 vaccine among people of a wide range of ages living in different countries. However, there were limited data available for assessing the effects of certain parameters (such as the effect of the dosing interval on post-dose two efficacy) on vaccine efficacy, which begat the need to conduct the post hoc exploratory analysis presented here.

\section{Conclusion}

This analysis demonstrates that in scenarios where the number of vaccine doses available is insufficient for the highest risk groups ( $>65$ years of age) to receive two vaccine doses, allocation of a single vaccine dose to twice the number of individuals or extending the time interval between doses may be more optimal strategies. In contexts without supply constraints, or if the single-dose efficacy is low, providing a booster dose would be preferable. Apart from these specific conditions, the results indicate that providing individuals with two doses of vaccine would have a similar effectiveness to the use of a single dose in twice the number of individuals. In an ideal world, decisions about vaccination strategies would be made within the exact parameters of the trials that have been 
conducted. However, the limited availability of resources, and specific country contexts, may require decision-makers to consider alternative strategies. 
Tables and Figures

Table 1: Model parameters.

\begin{tabular}{|c|c|c|c|}
\hline Parameter & Model term & Range & Description \\
\hline $\begin{array}{l}\text { Population attack size (\% of } \\
\text { the population) }\end{array}$ & ATT & $(4 ; 12 ; 20)$ & $\begin{array}{l}\text { This is the percentage of the population } \\
\text { infected within the 6-month study period }\end{array}$ \\
\hline $\begin{array}{l}\text { Vaccine allocation (\% of the } \\
\text { population during study } \\
\text { period) }\end{array}$ & TRG & $(5 ; 10 ; 20 ; 30)$ & $\begin{array}{l}\text { Allocation range was based on the assumed } \\
\text { administration speed. Using current data, } \\
\text { we assumed that higher-income countries } \\
\text { could reach a maximum speed, allowing } \\
30 \% \text { coverage of the population within } 6 \\
\text { months }\end{array}$ \\
\hline $\begin{array}{l}\text { Second dose administered } \\
\text { (\% of the vaccinated } \\
\text { population administered a } \\
\text { second dose) }\end{array}$ & DSP & $\begin{array}{c}(0 ; 25 ; 50 ; 75 ; \\
100)\end{array}$ & $\begin{array}{l}\text { This is the percentage of the vaccinated } \\
\text { population that are administered a second } \\
\text { (booster) dose }\end{array}$ \\
\hline $\begin{array}{l}\text { Interval between first dose } \\
\text { and booster dose }\end{array}$ & BTI & $\begin{array}{c}\text { (4 weeks; } 7 \\
\text { weeks; } 12 \text { weeks) }\end{array}$ & $\begin{array}{l}\text { The interval between doses can affect } \\
\text { vaccine efficacy; the range chosen was } \\
\text { based on available clinical trial data }\end{array}$ \\
\hline Vaccine delivery speed & DEL & $\begin{array}{l}\text { (fixed; } \\
\text { frontloaded; } \\
\text { backloaded) }\end{array}$ & $\begin{array}{l}\text { The speed of vaccine delivery to the } \\
\text { population - see Supplementary figure } 6\end{array}$ \\
\hline $\begin{array}{l}\text { Vaccine efficacy after the } \\
\text { second dose }\end{array}$ & PD2 & $(65 ; 75 ; 85)$ & $\begin{array}{l}\text { Maximum efficacy following the second } \\
\text { dose }\end{array}$ \\
\hline $\begin{array}{l}\text { Vaccine efficacy of the first } \\
\text { dose compared with the } \\
\text { second dose (\%) }\end{array}$ & D2B & $(50 ; 75 ; 100)$ & $\begin{array}{l}\text { Effect of the first dose compared with the } \\
\text { second dose }\end{array}$ \\
\hline $\begin{array}{l}\text { Immunity wane rate (days } \\
\text { following last dose) }\end{array}$ & VCW & $\begin{array}{c}(90 ; 180 ; 360 ; \\
540)\end{array}$ & Vaccine protection decay post-last dose \\
\hline
\end{tabular}


Fig. 1: Overall sensitivity analysis of vaccine effectiveness, based on UK data.

Deaths

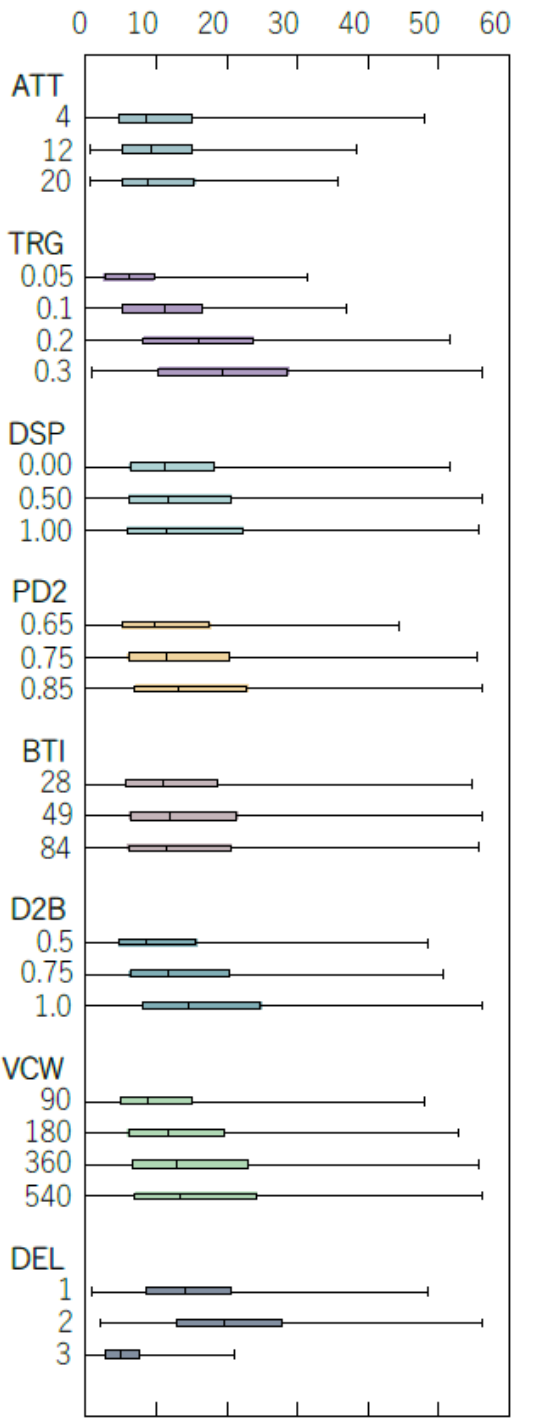

Clinical Cases
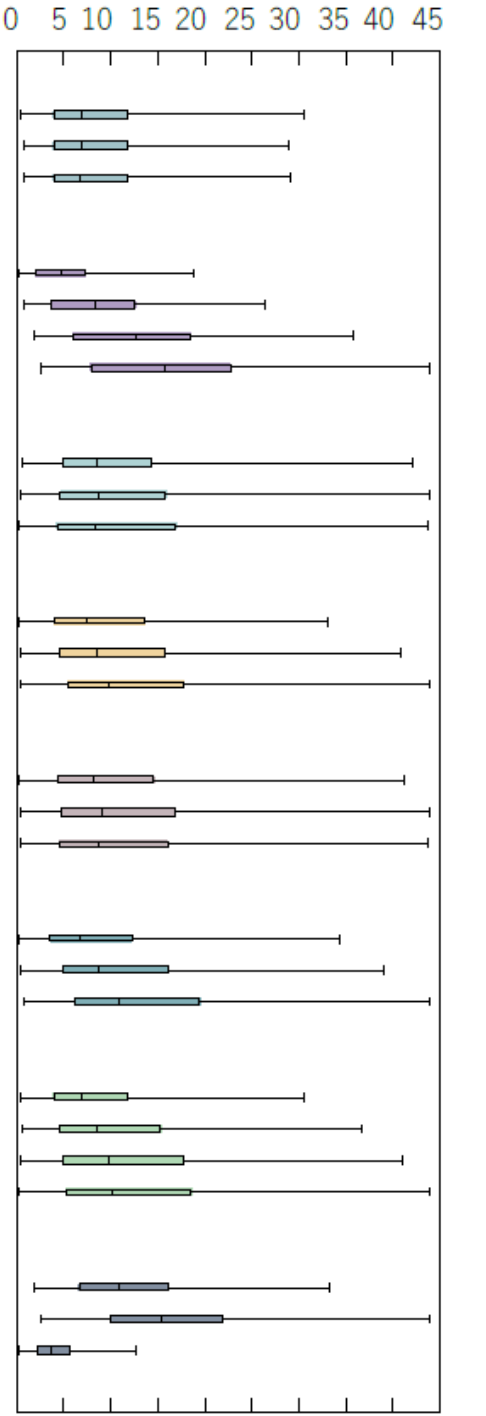

Infections

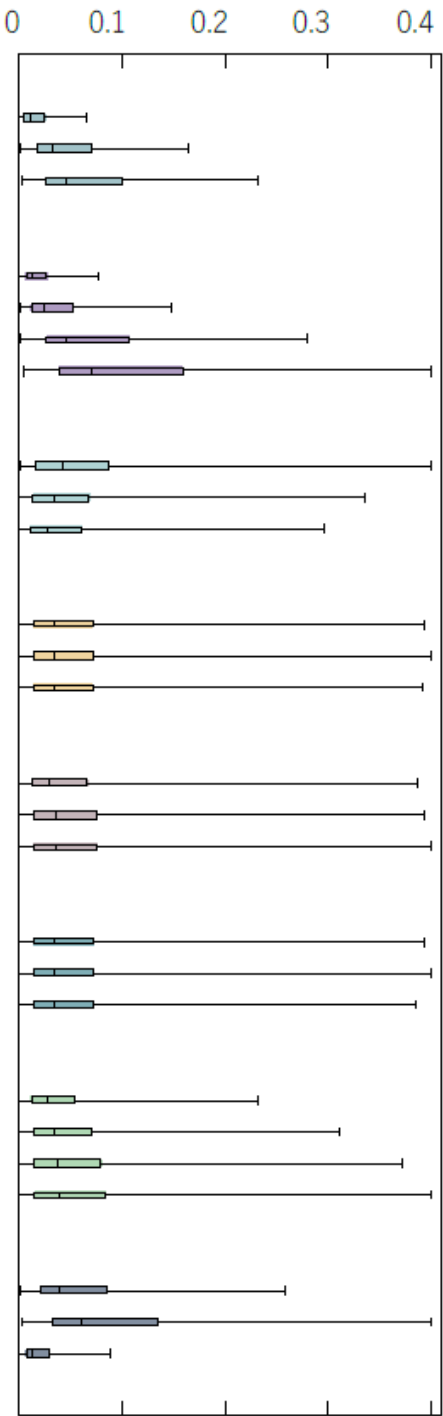

The boxplots show the median and interquartile ranges of the predicted vaccine effectiveness on each of the outcomes for specific parameters. They were generated by aggregating all model simulations for each of the parameters, with each boxplot summarizing the variance in predicted vaccine efficacy for all possible combinations of the other parameters. The full list of parameters explored and their descriptions can be found in Table 1. 
Fig. 2: Optimal dose allocation.

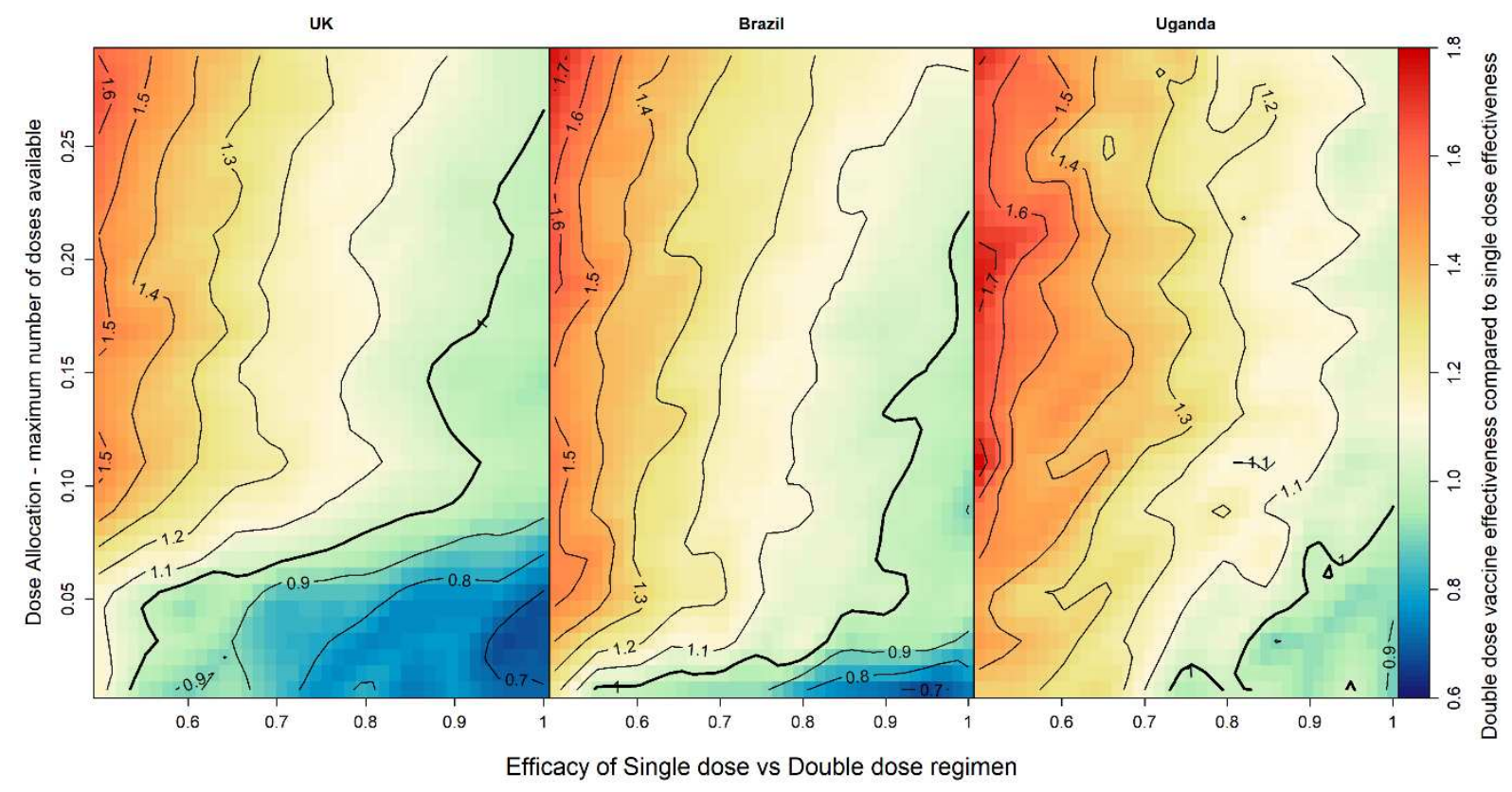

The coloured surfaces and respective contour lines indicate the ratio between the predicted vaccine effectiveness for a double-dose regimen vs a single-dose regimen. This ratio is a mean ratio, obtained by averaging out the ratios obtained in all model runs assuming the corresponding $\mathrm{x}$ and $\mathrm{y}$ parameter values and thus are not expected to be regular. Contour line 1 (thicker black line) indicates the parameter combinations for which there is no expected difference between giving everyone a single dose vs giving everyone two doses. For values greater than 1 (hot colours), a two-dose regimen is preferable, and for values less than 1 (cold colours), a single-dose regimen is preferable. 
Fig. 3: Dose allocation thresholds in different countries.

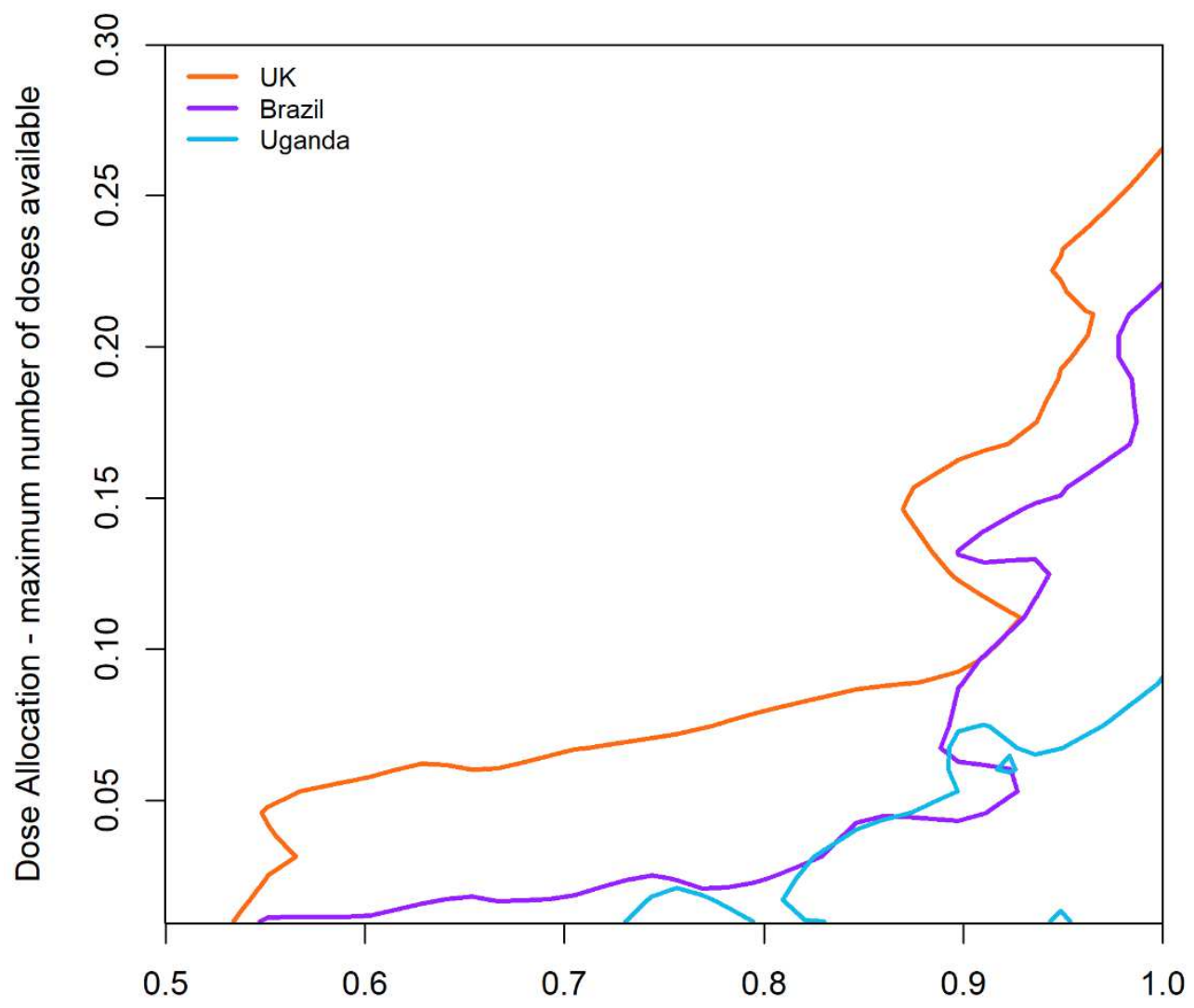

Efficacy of Single dose vs Double dose regimen

The figure illustrates the parameter combinations that define the allocation threshold above which a two-dose regimen would be preferred over a single-dose regimen. The areas under the curves are $16.5 \%, 8 \%$, and $3.8 \%$ for the UK, Brazil, and Uganda, respectively, which correlates almost perfectly with the proportion of the population above the age of 65 years in those countries. 
Supplementary Fig. 1: Impact of vaccine delivery speed, dose split, and dose availability on vaccine effectiveness as a measure of reduction in clinical cases.
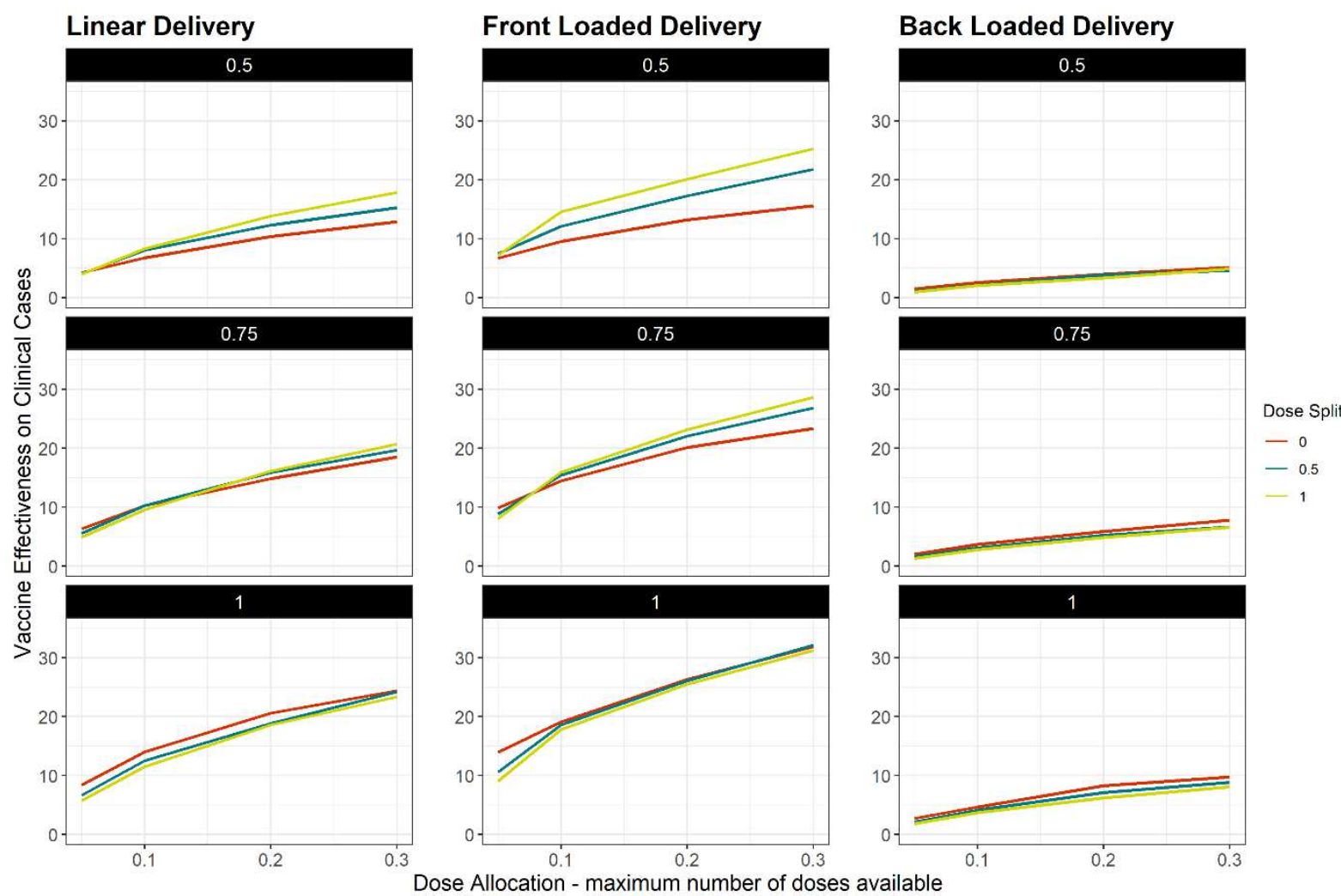

The white number on the black background in each panel defines the vaccine efficacy of the first dose relative to the second dose. Lines represent the mean effectiveness calculated using all runs, where the parameters are those defined by each figure, irrespective of all remaining parameters. Lines are coloured according to the dose split, i.e. the proportion of individuals receiving two vaccine doses. These results are based on the UK population structure. 
Supplementary Fig. 2: Impact of vaccine delivery speed, dose split, and dose availability on vaccine effectiveness as a measure of reduction in deaths.
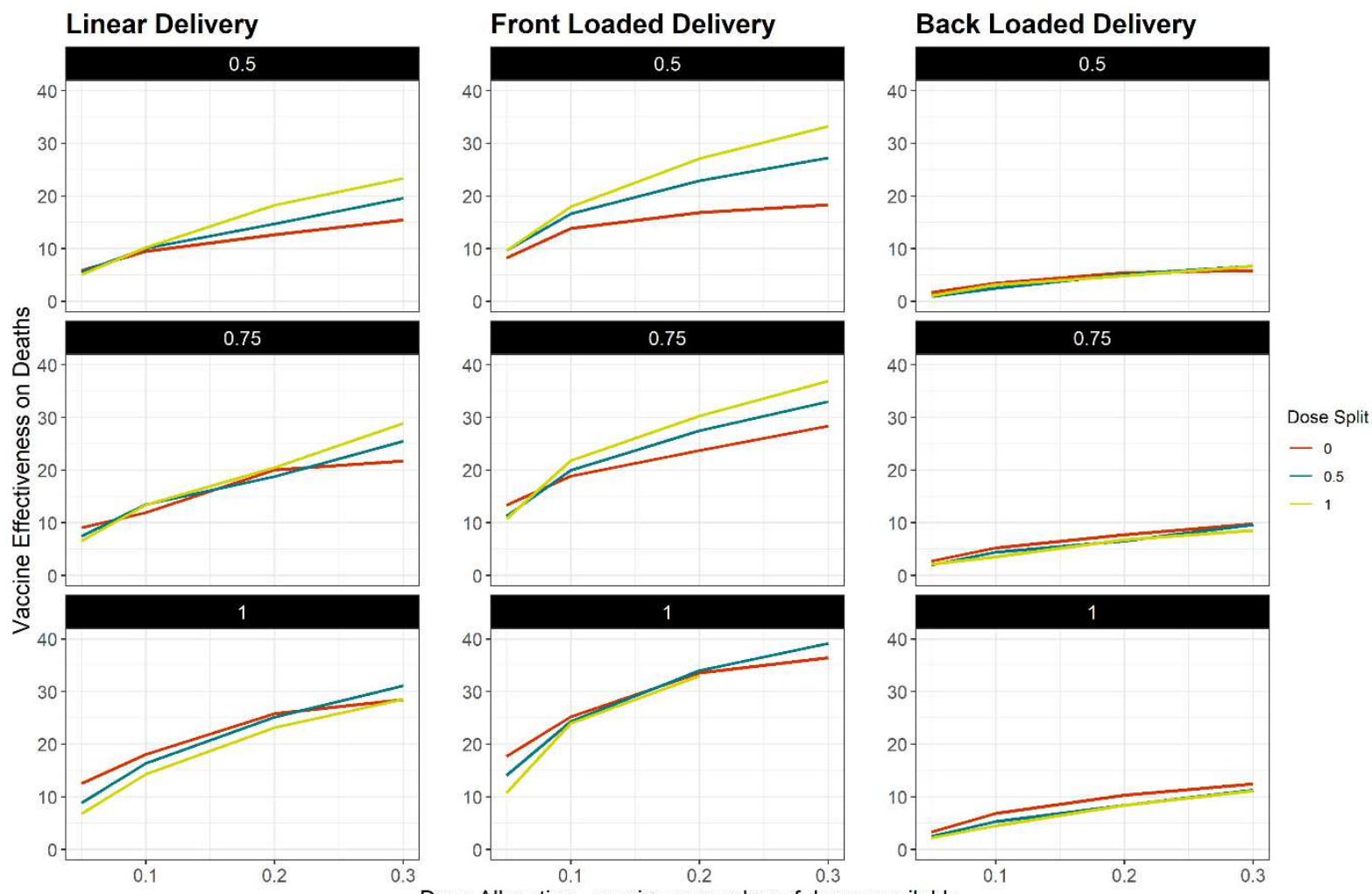

Dose Allocation - maximum number of doses available

The white number on the black background in each panel defines the vaccine efficacy of the first dose relative to the second dose. Lines represent the mean effectiveness calculated using all runs, where the parameters are those defined by each figure, irrespective of all remaining parameters. Lines are coloured according to the dose split, i.e. the proportion of individuals receiving two vaccine doses. These results are based on the UK population structure. 
Supplementary Fig. 3: Detailed sensitivity analysis of vaccine effectiveness for the most sensitive parameters, based on UK data.

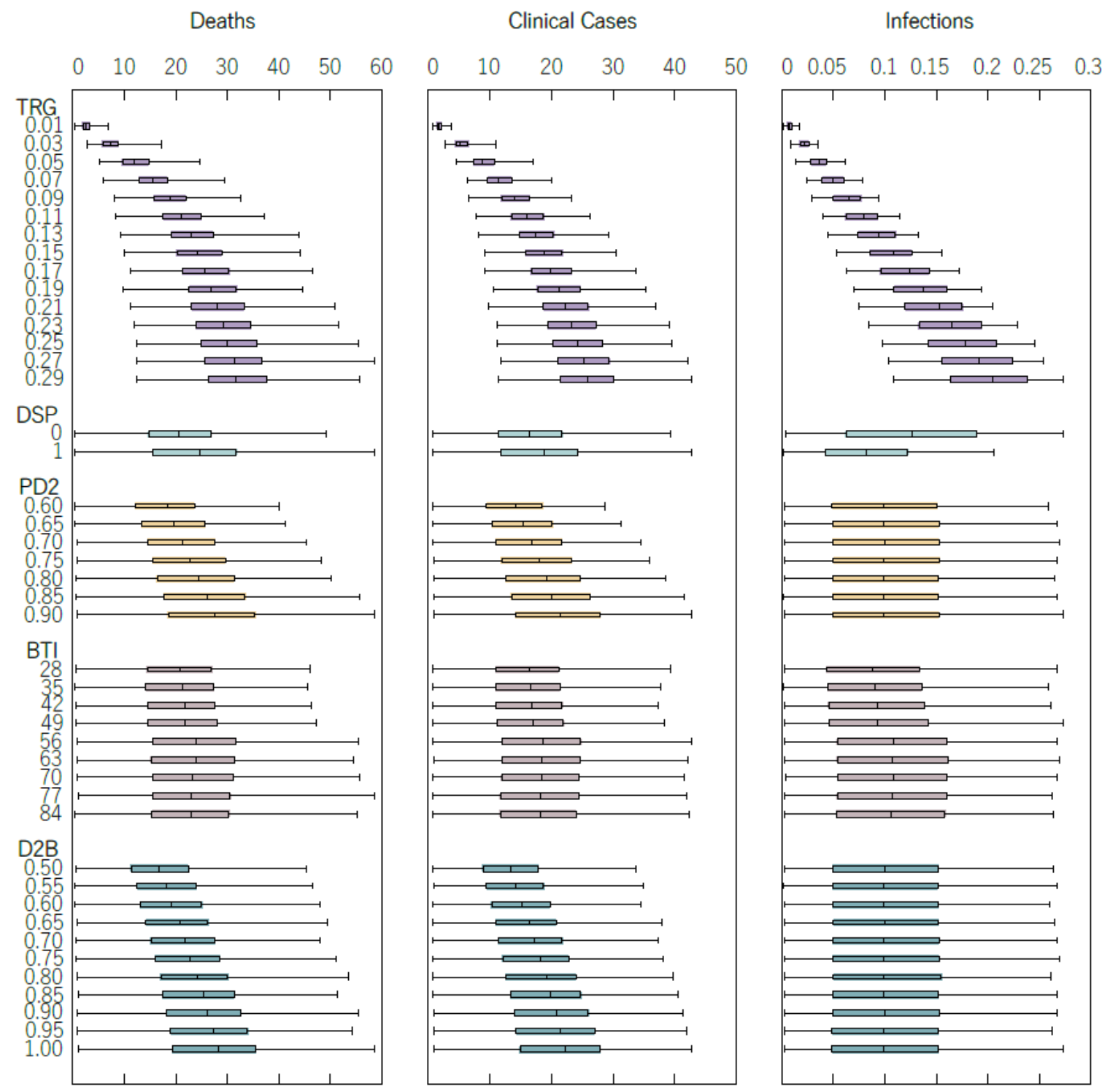

The boxplots show the median and interquartile ranges of the predicted vaccine effectiveness on each of the outcomes for specific parameters. They were generated by aggregating all model simulations for each of the parameters, with each boxplot summarizing the variance in predicted vaccine efficacy for all possible combinations of the other parameters. 
Supplementary Fig. 4: Impact of vaccine booster dose interval on vaccine effectiveness as a measure of reduction in clinical cases.
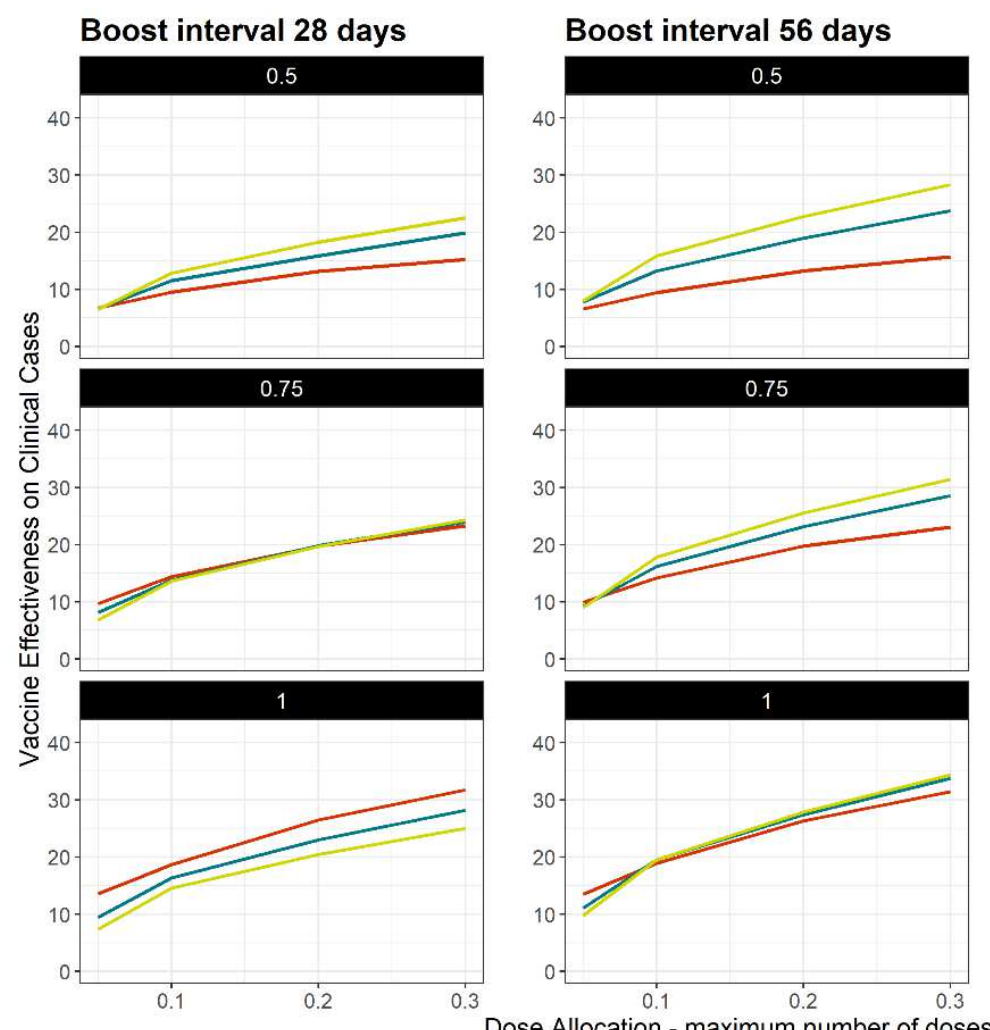

\section{Boost interval 84 days}
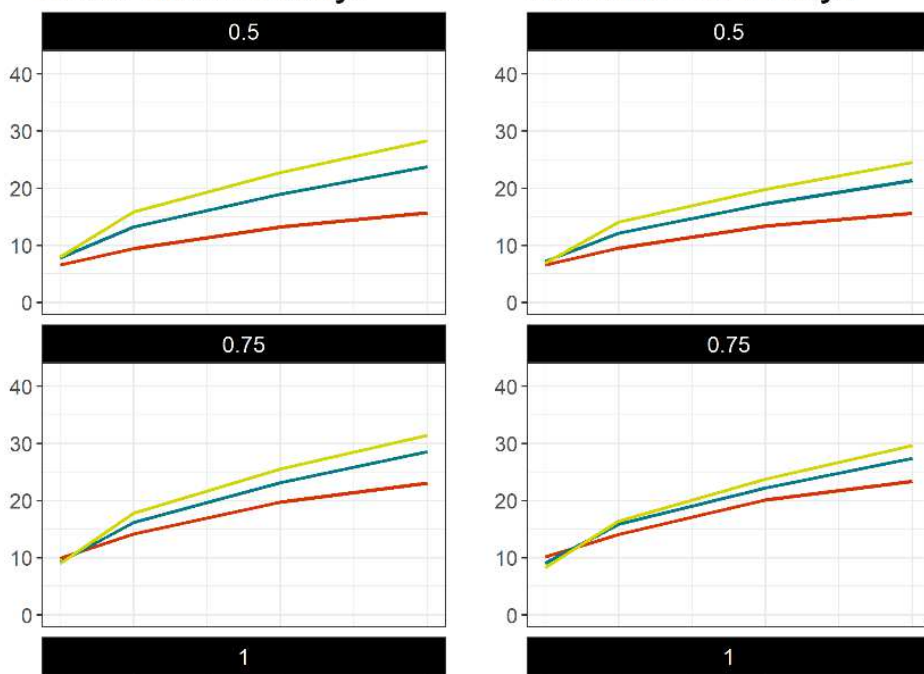

0.75
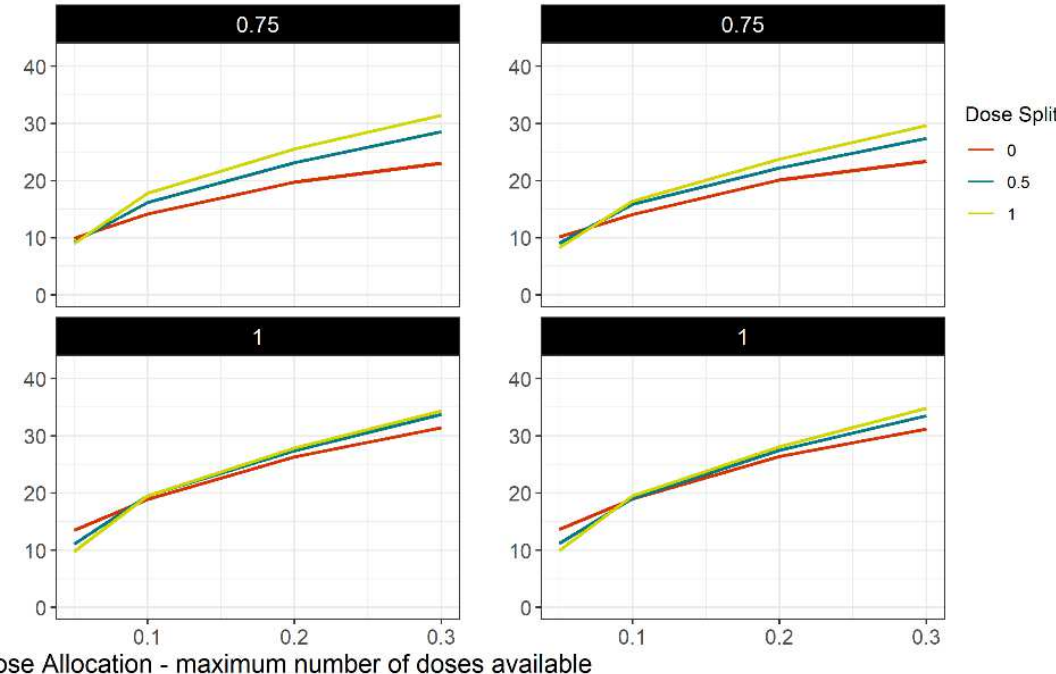

Dose Allocation - maximum number of doses available

The white number on the black background in each panel defines the vaccine efficacy of the first dose relative to the second dose. Lines represent the mean effectiveness calculated using all runs, where the parameters are those defined by each figure, irrespective of all remaining parameters. Lines are coloured according to the dose split, i.e. the proportion of individuals receiving two vaccine doses. These results are based on the UK population structure. 
Supplementary Fig. 5: Impact of vaccine booster dose interval on vaccine effectiveness as a measure of reduction in deaths.
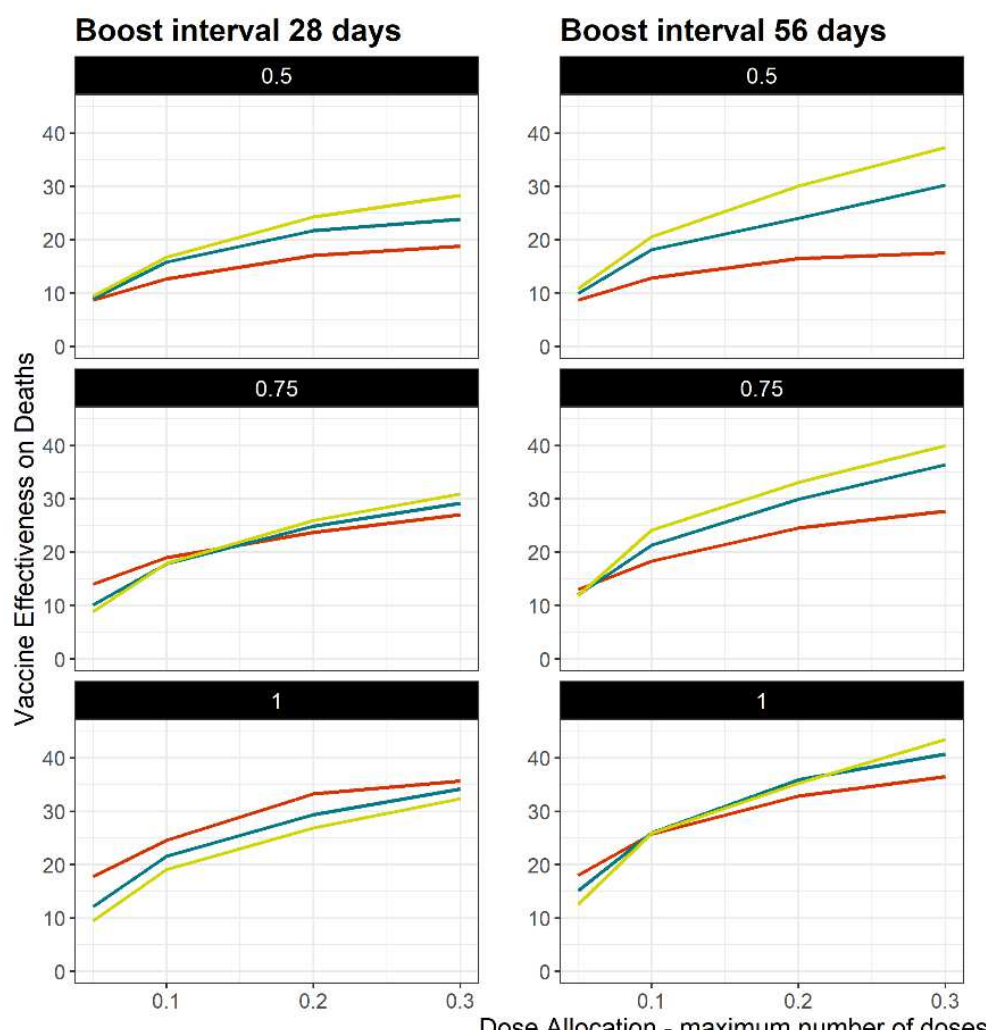

\section{Boost interval 84 days}
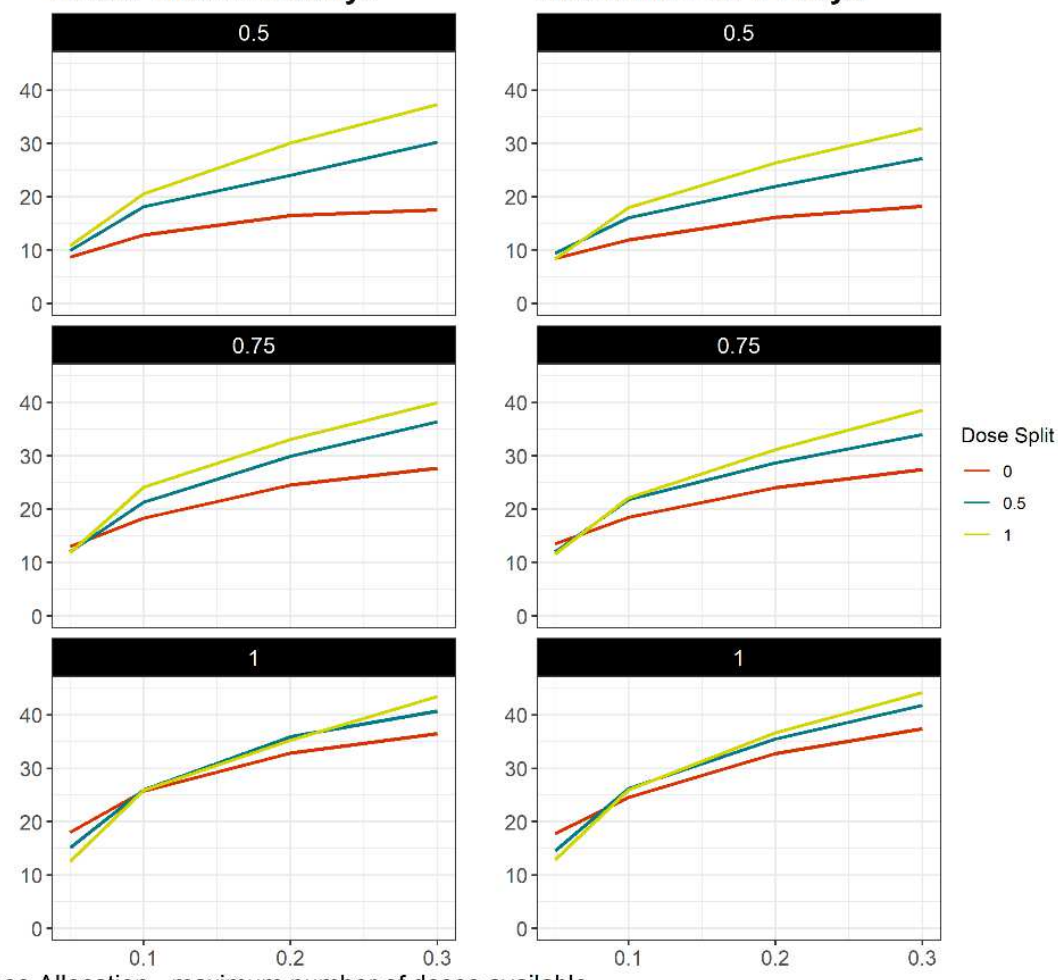

Dose Allocation - maximum number of doses available

The white number on the black background in each panel defines the vaccine efficacy of the first dose relative to the second dose. Lines represent the mean effectiveness calculated using all runs, where the parameters are those defined by each figure, irrespective of all remaining parameters. Lines are coloured according to the dose split, i.e. the proportion of individuals receiving two vaccine doses. These results are based on the UK population structure. 
Supplementary Fig. 6: Vaccine delivery speed.

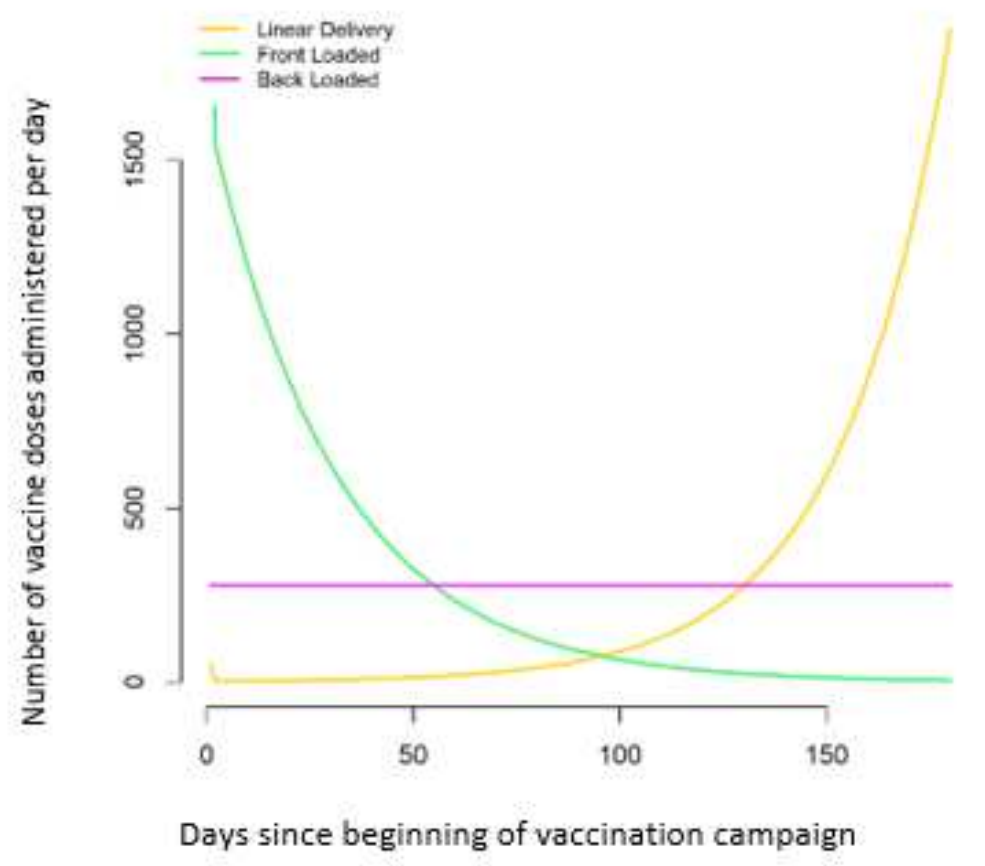

The figure shows the number of vaccine doses administered over the course of the vaccination campaign (6 months). 


\section{References}

1. Office for National Statistics. Coronavirus (COVID-19) weekly insights: latest health indicators in England, 12 February 2021. February 12, 2021 ed. United Kingdom; 2021.

2. Dong E, Du H, Gardner L. An interactive web-based dashboard to track COVID-19 in real time. Lancet Infect Dis 2020; 20(5): 533-4.

3. Johns Hopkins University. COVID-19 Dashboard. 2020.

https://coronavirus.jhu.edu/map.html (accessed June 2020.

4. Pfizer. Pfizer and BioNtech announce vaccine candidate against Covid-19 achieved success in first interim analysis from phase 3 study. 2020.

5. Folegatti PM, Ewer KJ, Aley PK, et al. Safety and immunogenicity of the ChAdOx1 nCoV-19 vaccine against SARS-CoV-2: a preliminary report of a phase $1 / 2$, single-blind, randomised controlled trial. Lancet 2020; 396(10249): 467-78.

6. Ramasamy MN, Minassian AM, Ewer KJ, et al. Safety and immunogenicity of ChAdOx1 nCoV19 vaccine administered in a prime-boost regimen in young and old adults (COVO02): a single-blind, randomised, controlled, phase 2/3 trial. Lancet 2021; 396(10267): 1979-93.

7. Voysey M, Clemens SAC, Madhi SA, et al. Safety and efficacy of the ChAdOx1 nCoV-19 vaccine (AZD1222) against SARS-CoV-2: an interim analysis of four randomised controlled trials in Brazil, South Africa, and the UK. Lancet 2021; 397(10269): 99-111.

8. MacLennan CA. Vaccines for low-income countries. Seminars in Immunology 2013; 25(2): $114-23$

9. Rodrigues CMC, Plotkin SA. Impact of Vaccines; Health, Economic and Social Perspectives. Frontiers in Microbiology 2020; 11(1526).

10. WHO. Fair allocation mechanism for COVID-19 vaccines through the COVAX Facility. Geneva: WHO, 2020.

11. Davies NG, Klepac P, Liu Y, Prem K, Jit M, Eggo RM. Age-dependent effects in the transmission and control of COVID-19 epidemics. Nat Med 2020; 26(8): 1205-11.

12. Pullano G, Valdano E, Scarpa N, Rubrichi S, Colizza V. Evaluating the effect of demographic factors, socioeconomic factors, and risk aversion on mobility during the COVID-19 epidemic in France under lockdown: a population-based study. The Lancet Digital Health 2020; 2(12): e638-e49.

13. Wang $\mathrm{W}, \mathrm{Wu} \mathrm{Q}$, Yang J, et al. Global, regional, and national estimates of target population sizes for covid-19 vaccination: descriptive study. BMJ 2020; 371: $\mathrm{m} 4704$.

14. lacobucci G, Mahase E. Covid-19 vaccination: What's the evidence for extending the dosing interval? BMJ 2021; 372: n18.

15. Mahase E. Covid-19: Medical community split over vaccine interval policy as WHO recommends six weeks. BMJ 2021; 372: n226.

16. Brazeau N, Verity R, Jenks S, et al. COVID-19 Infection Fatality Ratio Estimates from Seroprevalence. London: Imperial College London, 2020.

17. Voysey M, Clemens SAC, Madhi SA, et al. Single dose administration, and the influence of the timing of the booster dose on immunogenicity and efficacy of ChAdOx1 nCoV-19 (AZD1222) vaccine Lancet 2021.

18. Twohey M, Collins K, Thomas K. With First Dibs on Vaccines, Rich Countries Have 'Cleared the Shelves'. New York Times. 2020 December 15, 2020.

19. UN News. COVID-19 vaccines: donors urged to step up funding for needy countries. New York: UN News; 2020.

20. Davis N. Vaccine experts call for clarity on UK's 12-week Covid jab interval. The Guardian. 2021 January 25, 2021. 


\section{Figures}
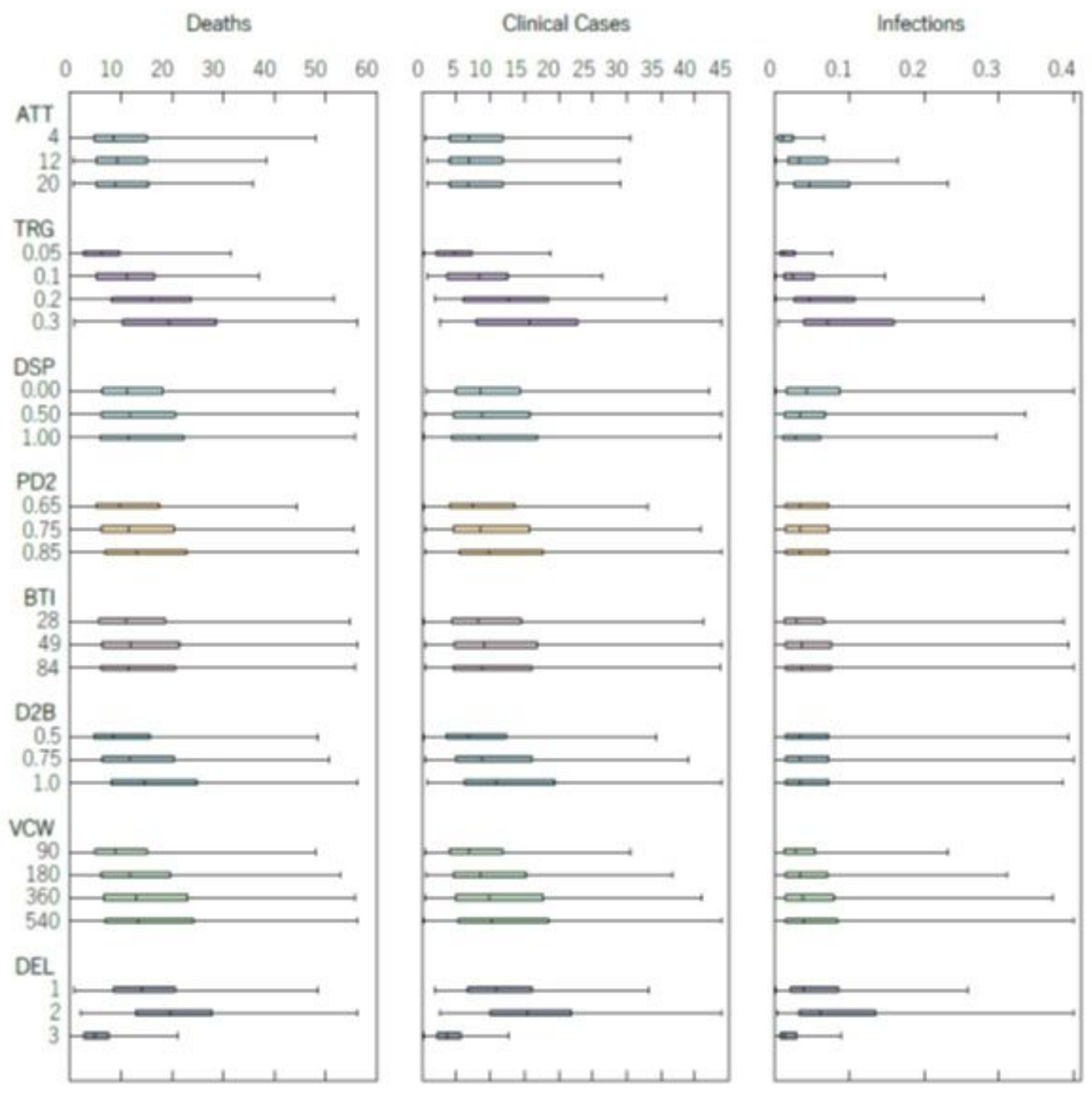

Figure 1

Overall sensitivity analysis of vaccine effectiveness, based on UK data. The boxplots show the median and interquartile ranges of the predicted vaccine effectiveness on each of the outcomes for specific parameters. They were generated by aggregating all model simulations for each of the parameters, with each boxplot summarizing the variance in predicted vaccine efficacy for all possible combinations of the other parameters. The full list of parameters explored and their descriptions can be found in Table 1 . 


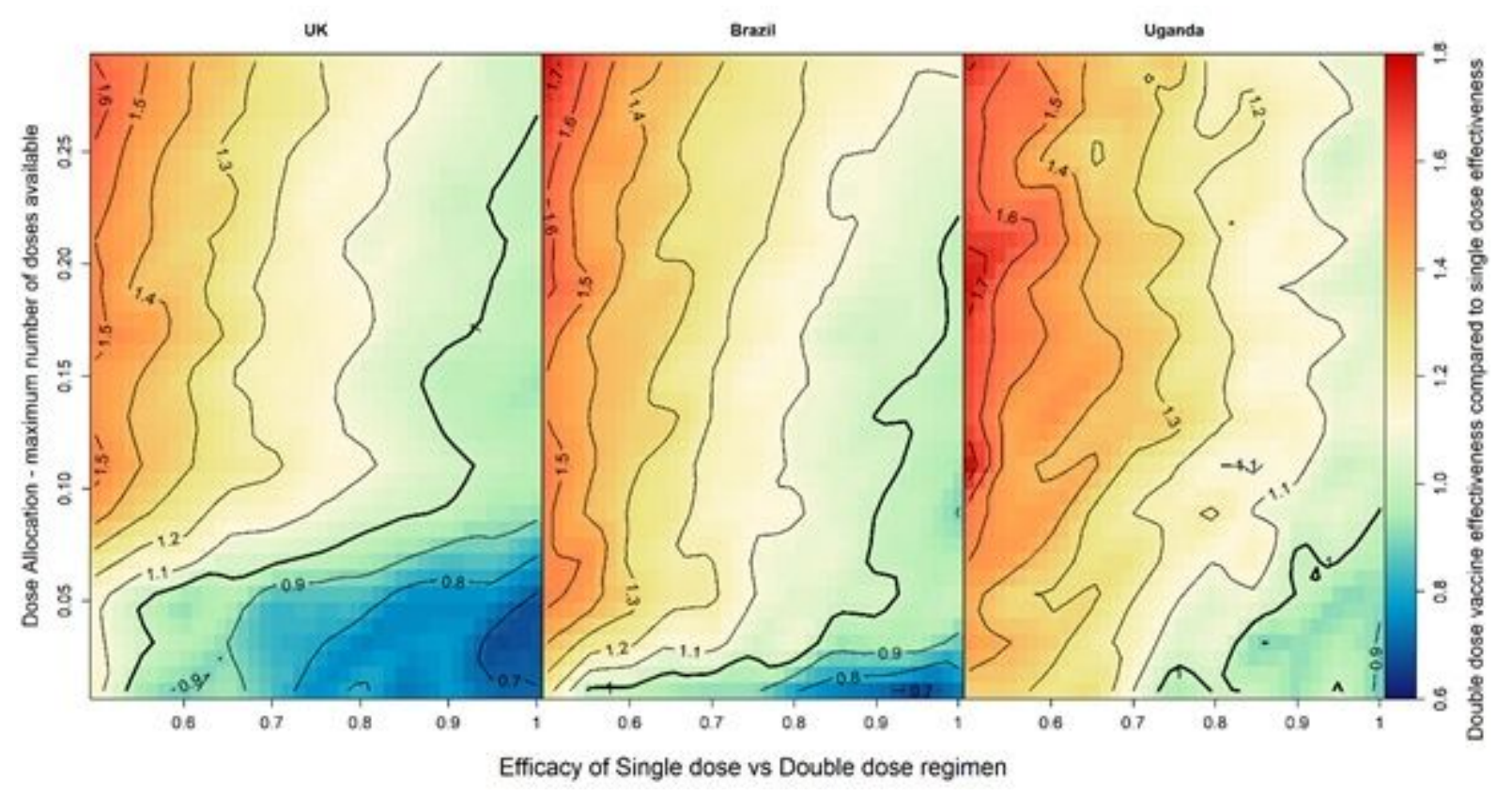

Figure 2

Optimal dose allocation. The coloured surfaces and respective contour lines indicate the ratio between the predicted vaccine effectiveness for a double-dose regimen vs a single-dose regimen. This ratio is a mean ratio, obtained by averaging out the ratios obtained in all model runs assuming the corresponding $x$ and y parameter values and thus are not expected to be regular. Contour line 1 (thicker black line) indicates the parameter combinations for which there is no expected difference between giving everyone a single dose vs giving everyone two doses. For values greater than 1 (hot colours), a two-dose regimen is preferable, and for values less than 1 (cold colours), a single-dose regimen is preferable. 


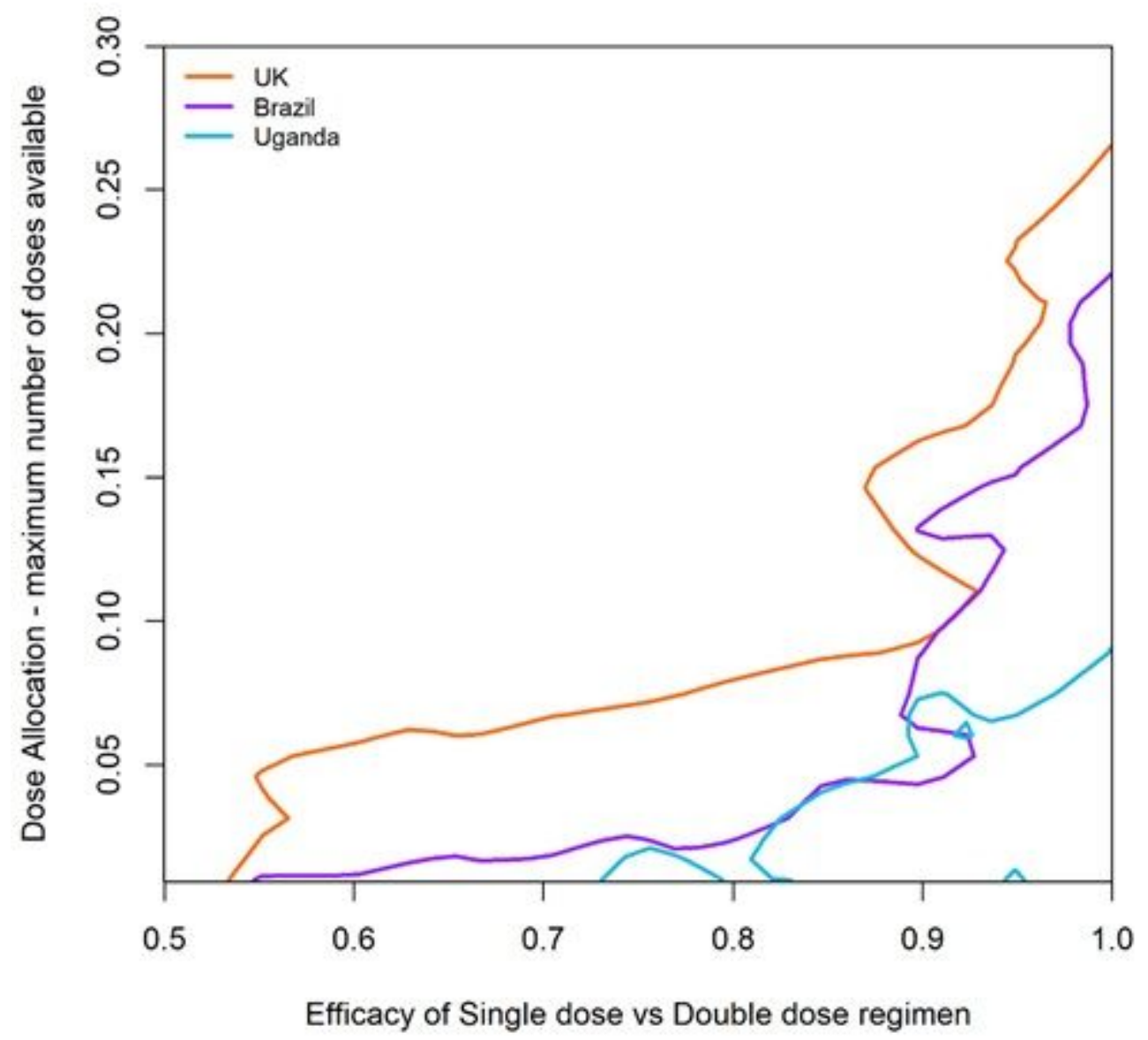

\section{Figure 3}

Dose allocation thresholds in different countries. The figure illustrates the parameter combinations that define the allocation threshold above which a two-dose regimen would be preferred over a single-dose regimen. The areas under the curves are $16.5 \%, 8 \%$, and $3.8 \%$ for the UK, Brazil, and Uganda, respectively, which correlates almost perfectly with the proportion of the population above the age of 65 years in those countries.

\section{Supplementary Files}

This is a list of supplementary files associated with this preprint. Click to download.

- SupplementaryFigures.pdf 